\title{
Can animal-free research be the future of medical science?
}

Professor Merel RitskesHoitinga from Radboud University, the Netherlands, aims to improve biomedical and medical research, reduce the use of unnecessary anim studies by using better alternatives. Throughout her career she has researched and highlighted the disadvantages of animal studies, such as the substandard quality of results produced and lack of evidence for translatability to human clinical research. To improve be a paradigm shift in the way we think about animal research - are animal studies really alternatives? Current science animal-free alternatives, such as human stem cells derived organoids and computer relevant results for humans.

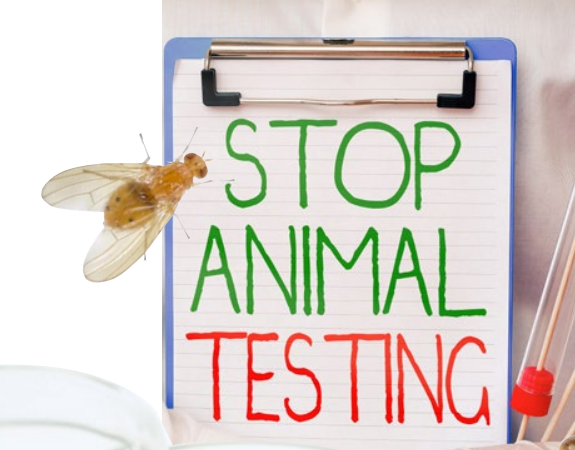

nimal models are non-human
species that are used in biological and biomedical research to find out more about human
life. From a clinical research standpoint, animal models are used by scientists aiming to discover the underlying causes of various diseases, preventative measures, and possible treatments. Using animal models can be traced back through millennia, when Aristot stated that studying animal species would enable better understanding of humans. Their use then became widespread during the $18^{\text {th }}$ and 19 centuries due to the expansion of medical science and have become a longstanding practice in research. But how useful is this practice?

The use of animal models to extrapolate information about to because of the conservation of certain all species. Some of the common models that are used include yeast, fruit flies (Drosophila), mice, rats, nematodes, frogs, zebrafish, and chick embryos. These models are chosen because of the considered close phylogenetic relationship to humans, which renders similarities in genetics, behavioural, and biochemical activities. For example, over $95 \%$ of genes are homologous (similar due to shared ancestry) between mice and humans. Between species many genes can be homologous, but this does not imply they will automatically respond to drugs have happer exple, disasters

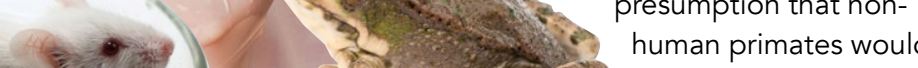

be good predictors of the response of the human immune system. The following advantages are considered when using animal models. important to study the pease, it is functions and syyte the physiological between organs and tissues. While cell cultures can be helpful in identifying molecular and cellular interactions, whole organisms are still considered necessary to elucidate the complex network of hormones, circulating factors, and cells. Progress in genetic engineering techniques in recent years also enabled developing diseased animal models with mutations or abnormal gene function through "knockout" studies, which is not possible to conduct in humans. However, the progress in genetic curing Alzheimer's disease, for curing Alzheimer's disease, for genetically modified models for this purpose is now strongly discouraged.

\section{DISADVANTAGES O} ANIMAL MODELS Despite the many advantages that animal models have brought us, such as analysing essential dietary nutrients, its use has become more and more a polarising point of discussion, based on new scientific evidence. In a 2020 . she ar Hoitinga and her colleagues, she argued for the increased use of animal-free research, such as computer modelling and organoids derived from human stem cells, as alternatives to animal models. As a veterinary medica
student three decades ago, Prof

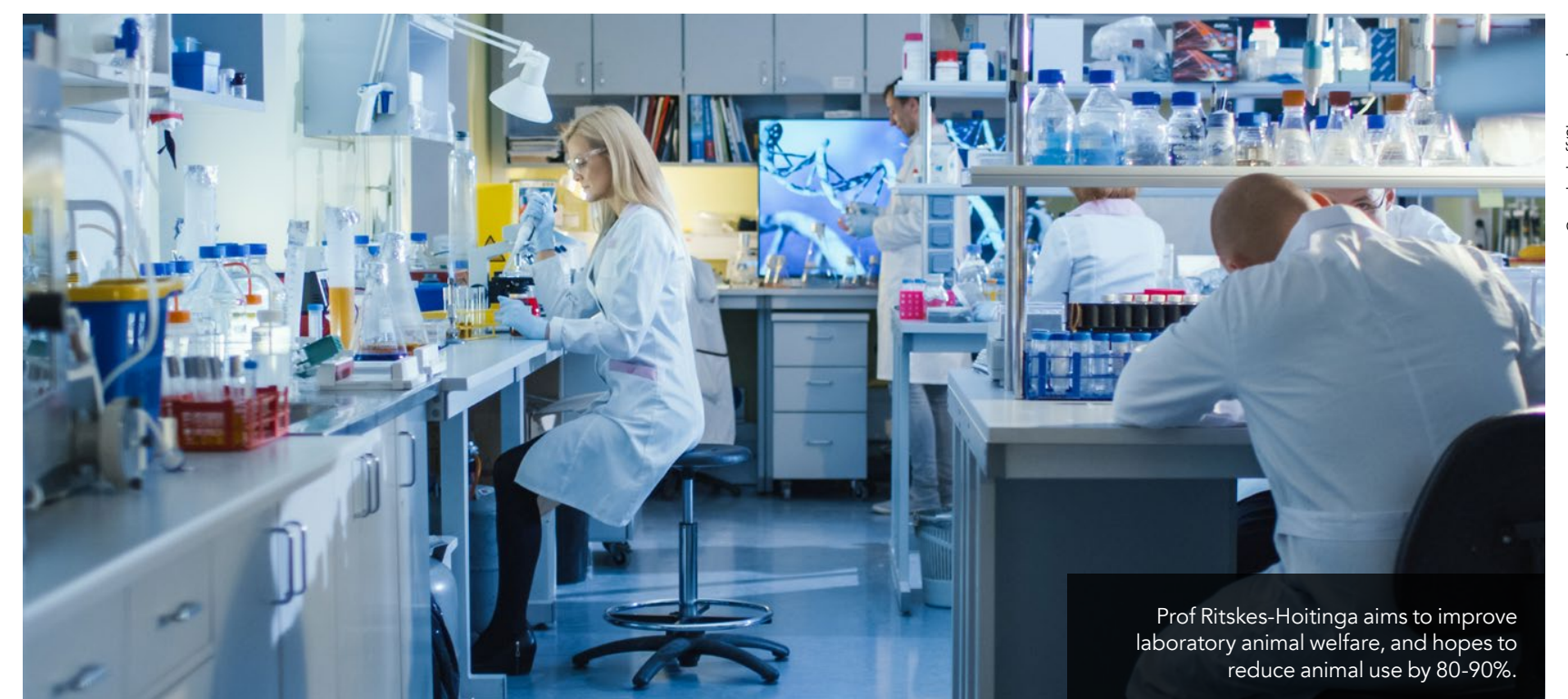

Ritskes-Hoitinga aspired to advance the qually of anmal research and witnessing the low quality of many published scientific papers. The resuts in these substandard papers could not be extrapolated to human-based trials, therefore many animal models were used without a clear medical purpose.

Recently, it was found that the results from only $25 \%$ of animals used in

research were considered substantia enough to be reported in papers. As a general rule, all results of all in relis need to be reported to result the $25 \%$, more

than half were of poor quality as themselves were performed were performed
inadequately

or not properly reported. All in all, it was found that in total, only about $10 \%$ of animal research was useful. This also has had a negative knock-on effect for the pharmaceutical industry first wish to repeat already published preclinical animal-based experiments themselves, to verify results before testing on humans. In a study by pharma, two thirds of the repeat studies gave inconsistent results with ealler published results, leading to disconthuation of these projects. Such hon-reproducibility of animal research billion annually in the USA alone. Moreover, "knockout" animal models may be advantageous to simulate mutation a specific genetic background. However it does not provide a holistic understanding of patients, as they usually have comorbid diseases and a disease is often not caused by a single gene but the meraction of several different ones, factors. In many experiments, animal populations are standardised to produce homogenous sample sizes and kept in regulated conditions, whic .in many cases, animal models fail to eplicate complex human behaviour and diseases.

of human populations. Therefore, in many cases, animal models fail to replicate complex human behaviour and diseases, producing inconsistent results when applied to humans in potential pharmacological interventions from animal models fail to recapitulate the same payoff in human clinical trials. Therefore, of the several thousand 500 ares that affect humans, only about 500 are estimated to have at least one approved treatment. Thus, the lab to bedside is in a crisis. A review of the publication dates of animal and human studies for the that animal and human-based research often concurrently occur rather than consecutively. The law requires animal studies to be conducted prior to clinical trials for safety testing. Therefore, how necessary is animal research really to egulations that calls for its widespread before clinical trials?

HOW DO WE IMPROVE PRECLINICAL RESEARCH? Because of the current evidence of lack

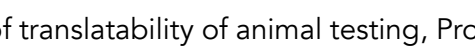
Ritskes-Hoitinga is advocating towards use by $80-90 \%$. She is aspiring to use less higher quality results, animal-free methods, with the aim of improving translation and more human-relevant science.

To overcome the lack of translatability of animal research to human clinical trials, Prof Ritskes-Hoitinga argues for a paradigm shift in the way we design and perform preclinical research. For thromple, increased transparency no experiment the results can been conducted and all hesesuls can be correcly interpreted. also be published and opuls should also be published and openly accessible 


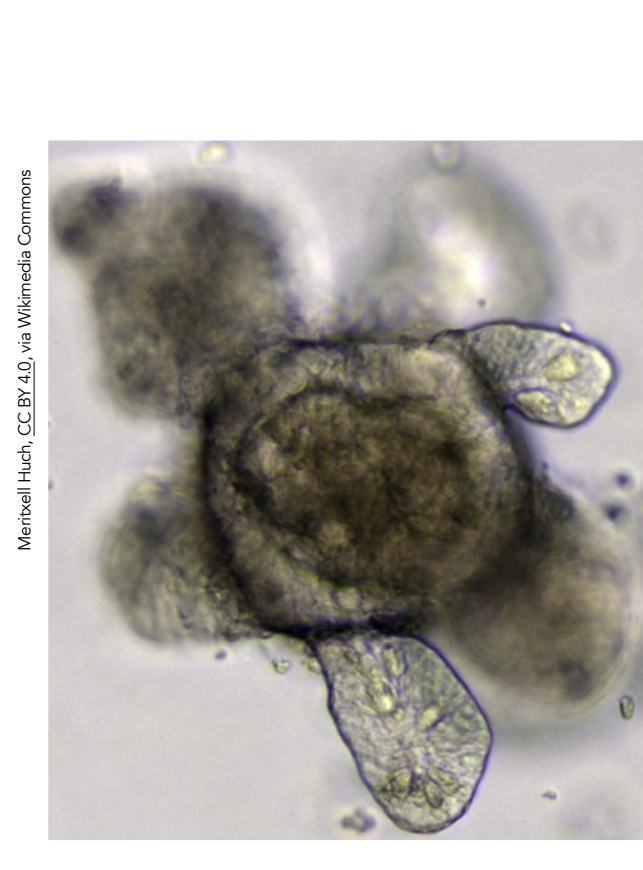

human stem cell derived organoids

lad collaborative culture has to exist, such as building open databases, and adoption of a replication culture to ensure that clinical research is continually and critically appraised. Research should also be carefully planned by involving relevant stakeholders, such as patients and patient organisations, leading to relevant medical research questions.

In addition, prior to conducting clinical research on human participants, a prospective systematic review (SR) of animal study results can be conducted. A systematic review scrutinises past experiments involving animal research strength of experio ental design methodof experimental design, summarise large volumes of evidence and analyse internal and external validity (accuracy of results in the studied population and accuracy of results in other situations, for example, generalisation and translatability
of findings from animal models to humans, respectively), which ultimately determines the safety and efficacy of proceeding with a trial.

Since conducting SRs of animal studies, Radboud University in the Netherland has decreased the use of animals in research by $35 \%$. Systematic reviews can reduce the use of unnecessary animal

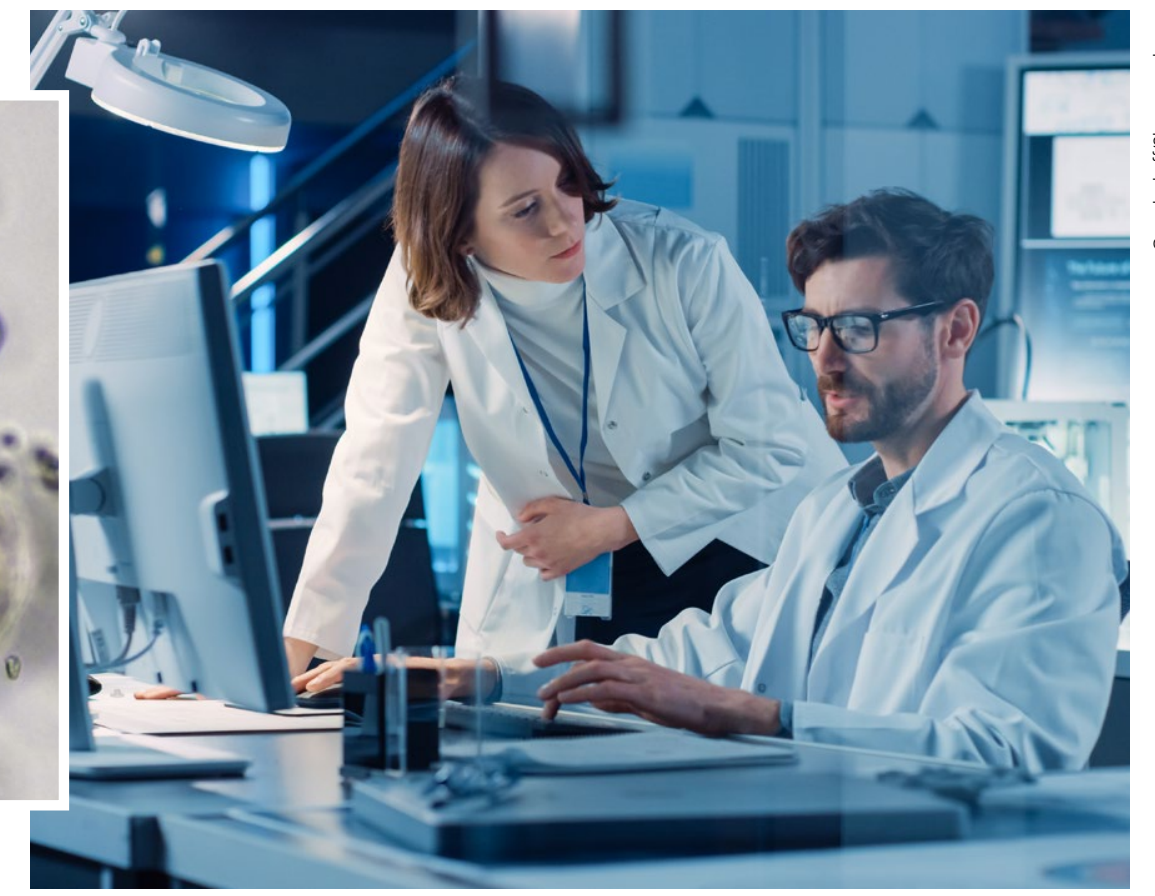

ongoing research to select more optimal animal models or animalfree alternatives. Systematic reviews criticaly summarise already existing evidence, and can thus provide new evidencebased insights for researchers to decide the next steps forward. This can preve dication of animal studies, avoid selecting inappropriate of clinical trials.

The unfortunate benefit of systematic reviews is that it has made clear that many animal experiments did not incorporate essential procedures that of results and trans rabrilisy

couraged more discussions and wareness amongst researchers to utilise existing guidelines for experimentations. that the quality of experimental design is upheld.

In conclusion, the use of animal models has to undergo continuous scrutiny to ensure no animals are used unnecessarily. The current structure of research utilises many animals in preclinical research but fails to produce significant results when translating to human clinical trials. Therefore, humancentred models, such as the use of organoids derived from human stem get priority. With continuing advocacy

Polarisation surrounding the pros and cons of animal testing for medical science can be solved by analysing the scientific evidence through the undertaking of systematic reviews. the execution of the basic scientific principles of randomisation and blinding. Therefore, the increased use of systematic reviews can potentially foreground his issue and be a catalyst for the improvements of experim design, quality, and reporting. and direct collaboration with multiple stakeholders, such as politicians and patient organisations, Prof RitskesHoitinga aspires to spearhead the nnovation of animal-free research in the standard of biomedical research globally,
benefitting both animals and humans. Netherlands and hopefully improve the

\section{Behind the Research}

\section{Prof Merel Ritskes-Hoitinga}

E: Merel.Ritskes-Hoitinga@Radboudumc.nl T: $+31653519387 \quad$ W: www.syrcle.nl

\section{Research Objectives}

Professor Merel Ritskes-Hoitinga encourages more evidence-based high quality preclinical research, leading to Reduction and Replacement of animal testing and more human relevant science.

\section{Detail}

Address Prof Ritskes-Hoiting

SYRCLE, Radboudumc, Nijmegen, The Netherland Bio

Dr Merel Ritskes-Hoitinga is Professor in EvidenceBased Laboratory Animal Science, Department for Health Evidence, Radboud University Medical Center. She also holds an Honorary SKOU professorship at the Department of Clinical Medicine, Aarhus University, Denmark.

Funding

- NWO

- ZonMw

- DG Environment Brussels

- Joint Research Center Ispra

Collaborators

- Evidence Based Toxicology Collaboration Value in Research Funder Forum - Evidence Synthesis International

- Cocr Medicinesoration

- AUGUST at Aarhus University Denmark

- Department of Comparative Medicine, Karolinska Institute, Sweden

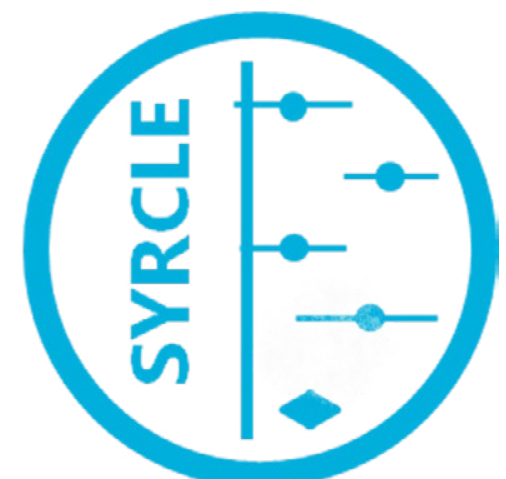

\section{References}

Ritskes-Hoitinga, M. (2021). Opinion: Animal-free alternatives lead to better science [online]. de

Volkskrant. https://www.volkskrant.n//columns-opinie/ opinie-proefdiervrije-alternatieven-leiden-tot-beterewetenschap b9def180/

Ritskes-Hoitinga, M., Leenaars, C., Beumer, W., Roo, T. C. et al. (2020). Improving translation by identifying evidence 10,1170

Pound, P. \& Ritskes-Hoitinga, M. (2020). Can prospective systematic reviews of animal studies improve clinical translation? Journal of Translational Medicine, $18,15$.

Ritskes-Hoitinga, M. (2019). Delivering better evidence with non-animal studies. Inaugural lecture transcript. Presented at Systematic Review Center for Laboratory Animal Experimentation (SYRCLE). 06 Nov.

Pound, P. \& Ritskes-Hoitinga, M. (2018). Is it possible to overcome issues of external validity in preclinical animal research? Why most animal models are bound to fail.

\section{Personal Response}

Why is it important to collaborate with different stakeholders, such as polticicians, to advance your issue of animal-free research?

Despite decades of widespread scientific evidence demonstrating the lack of quality of publications on animal studies and the lack of translatability, the feientimp wovements. Thereere it is necessary to fast improvements. Therefore, it is necessary to
involve other stakeholders such as politicians and patient organisations, in order to make sure that the necessary improvements indeed become implemented in scientific practice. The lack of translation of many paradigm. Politicians can play a crucial $r$ a inferent the necessary change by making much more funding available for animal-free research. Moreover, politics is needed to change the routine of legally required animal studies for drug and vaccine development towards a new paradigm. This will be of great benefit for both 